\title{
Estrogen Receptor-1 Genetic Polymorphisms for the Risk of Premature Ovarian Failure and Early Menopause
}

\author{
Jae Jeong Yang, M.P.H., ${ }^{1}$ Lisa Y. Cho, M.P.H., Y Yun Jeong Lim, M.D., Ph.D., ${ }^{2}$ \\ Kwang-Pil Ko, M.D., Ph.D., ${ }^{1,3}$ Kun-Sei Lee, M.D., Ph.D., ${ }^{4}$ Hyeongsu Kim, M.D., Ph.D., ${ }^{4}$ \\ Sung Vin Yim, M.D., Ph.D., 5 Soung Hoon Chang, M.D., Ph.D., ${ }^{4}$ and Sue K. Park, M.D., Ph.D., ${ }^{1,6}$
}

\begin{abstract}
Background: The aim of this study was to investigate the role of the estrogen receptor 1 (ESR1) genetic polymorphisms for early menopause that was classified as premature ovarian failure (POF) and early menopause (EM) and to examine whether the associations of ESR1 genetic variants are different for POF and EM.

Methods: We selected 100 POF cases and matched 100 EM cases and 200 normal menopause (NM) controls from the Korean Multi-Center Cohort. Among them, we restricted idiopathic POF and EM cases vs NM controls by excluding POF/EM cases with medical/surgical causes. The XbaI (rs9340799) and PvuII (rs2234693) in the ESR1 gene were genotyped. The single-nucleotide polymorphism (SNP) and haplotype effects were analyzed by multivariate logistic regression and haplotype analysis. Also nominal polytomous logistic regression was used to find whether ESR1 genetic variants are differently associated with POF and EM.

Results: The global $p$ values for idiopathic POF and EM were 0.08 and 0.39 (SNP-based), and $<0.001$ and 0.12 (haplotype-based), respectively. The $X b a \mathrm{I}$ genetic variant containing the $\mathrm{X}$ allele was marginally significantly associated with a reduced risk of idiopathic POF $(\mathrm{OR}=0.6,95 \% \mathrm{CI} 0.3-1.0)$. The P-x haplotype and diplotypes significantly decreased the risk of idiopathic POF $(\mathrm{OR}=0.5,95 \%$ CI $0.2-0.9$; OR $=0.4,95 \%$ CI 0.2-0.9, respectively). In contrast from POF, the P-x haplotypes and diplotypes insignificantly increased the risk for both idiopathic EM ( $p_{\text {polytomous }}=0.009$ for P-x haplotype; $p_{\text {polytomous }}=0.02$ for P-x diplotypes).

Conclusion: Our results suggest that the ESR1 gene including PvuII and XbaI polymorphisms may modify the risk of idiopathic premature ovarian failure (POF) but not idiopathic early menopause (EM) risk.
\end{abstract}

\section{Introduction}

A GE AT MENOPAUSE, as well as overall years of menstruation, has major implications for women's health. The time of menstruation cessation is an important contributing risk factor for postmenopausal diseases. ${ }^{1-5}$ In general, menopause at an early age implies greater susceptibility to various disorders. Previous studies reported that early menopause (EM) before the age of 45 was associated with a greater risk of osteoporosis, fractures, heart disease, cancer, and all-cause mortality. ${ }^{6-12}$ The definition of early menopause from previous studies, however, was a more inclusive definition that included both premature ovarian failure (POF) and early menopause, not exclusively POF.

POF is a disorder defined as the cessation of menstruation that occurs before the age of $40 .^{13}$ The exact mechanism of POF is unclear, but several hypotheses have been proposed, such as an abnormality of the ovary or of genetic function. ${ }^{14-16}$ In contrast, early menopause that occurs before the age of 45 years is assumed to be due to hormonal changes rather than a genetic effect. The etiology of these related conditions is unknown, because most previous studies do not distinguish

\footnotetext{
${ }^{1}$ Department of Preventive Medicine, Seoul National University College of Medicine, Seoul, Korea.

${ }^{2}$ Department of Internal Medicine, Dongguk University College of Medicine, Ilsan, Korea.

${ }^{3}$ Division of Epidemiology and Health Index, Center for Genome Science, Korea Center for Disease Control and Prevention, Seoul, Korea.

${ }^{4}$ Department of Preventive Medicine, Konkuk University School of Medicine, Chungju, Korea.

${ }^{5}$ Department of Clinical Pharmacology, Kyunghee University School of Medicine, Seoul, Korea.

${ }^{6}$ Seoul National University Cancer Research Institute, Seoul, Korea.

All the authors-Jae Jeong Yang, Lisa Y. Cho, Yun Jeong Lim, Kwang-Pil Ko, Kun-Sei Lee, Hyeongsu Kim, Sung Vin Yim, Soung Hoon Chang, and Sue K. Park-contributed to the design, conduct, and analysis of the study, the drafting and revision of the paper, and approved the final version of the paper.
} 
between POF and early menopause. Additionally, because of the broader definition of early menopause used in previous studies, the actual POF risk may be underestimated and misinterpreted. Therefore, we classified early menopause into two separate categories: POF and EM. As defined in previous studies, POF is menopause that occurs before the age of 40 , and EM is defined as menopause that occurs between ages 40 and $44 .^{17}$

Studies have investigated the association between genetic factors and age at menopause. ${ }^{18-28}$ Genetic polymorphisms of estrogen receptors (ERs) expressed by ER- $\alpha$ (ESR1/estrogen receptor 1$)$ and ER- $\beta$ (ESR2/estrogen receptor 2$)^{29}$ have been examined. ${ }^{21-28}$ The two most widely studied polymorphisms of ESR1 are XbaI and PuvII, which are located on the first intron 397 and $351 \mathrm{bp}$ upstream of exon 2, and have a high degree of linkage disequilibrium. ${ }^{30}$ Though many studies suggest that genetic polymorphisms of ESR1 modify susceptibility to women's disorders, including osteoporosis, endometriosis, preeclampsia, and breast cancer, few studies have demonstrated the association of ESR1 and the risk of POF and/or EM.

ESR1 genetic polymorphisms seem to play a role in developing POF and/or EM, but the exact mechanism is still unclear. Thus, we hypothesized that genetic variants of ESR1 underlie the association with POF and/or EM, and the effect of ESR1 in POF may differ from that of EM. In this study, we classified early menopause into two groups (POF and EM), and examined whether ESR1 genetic polymorphisms are associated with only POF or both POF and EM.

\section{Materials and Methods}

\section{Study population}

Our study population was selected from the Korean MultiCenter Cancer Cohort (KMCC), a prospective cohort of participants recruited from four urban and rural areas in Korea (Haman, Chungju, Uljin, and Youngil). The study protocol for the KMCC was approved by the institutional review boards of Seoul National University Hospital and the National Cancer Center of Korea (H0110-084-002). Detailed information about the KMCC study has been described elsewhere. ${ }^{17,31}$ We chose four districts from Chungju, one of the local centers, and performed community surveys. To maximize the participation rate, additional telephone surveys were also performed for women who did not participate in the community survey. A total of 2,668 women between the ages of 30 and 79 were recruited; the participation rate was $70.2 \%$. We included postmenopausal women $(n=1,919)$ and excluded subjects over age $70(n=51)$ because of recall bias and subjects with no information on their age at menopause. $(n=132)$. Menopause was defined as a period of amenorrhea greater than 12 consecutive months. We defined three menopausal groups: POF $(n=137)$ was defined as cessation of menstruation before age 40; EM was defined as menopause between the ages of $40-44$ years $(n=281)$; normal menopause (NM) controls $(n=1,318)$ were defined as menopause between the ages of 45-60. Idiopathic POF and EM were defined as POF and EM not due to any medical causes or relevant surgical causes for cessation of menstruation. Fifty-three women from the POF group and 20 from the EM group had surgical or medical menopause related to hysterectomy, oophorectomy, cancer therapy, and various other hormonal diseases. Eighty-four idiopathic POF and 261 idiopathic EM cases were identified from our eligible population. After excluding subjects without blood samples, 103 POF and 187 EM subjects remained. We matched one POF case to one EM case and two NM controls, according to age $(\leq 60,>60$ years old) and the number of years from menopause $(\leq 10,>10$ year). Finally, we selected 100 POF cases, 100 EM cases, and 200 NM controls, including 46 idiopathic POF and 86 idiopathic EM cases.

\section{Data collection}

All participants signed a consent form and completed a detailed standardized interview-based questionnaire that included information on demographic characteristics, family history, medical history, reproductive factors, use of oral contraceptives, physical activity, use of agricultural chemicals, cigarette smoking, alcohol consumption, and other environmental risk factors.

Genomic DNA was prepared from whole blood samples using the genomic DNA purification kit (Core-One ${ }^{\mathrm{TM}}$ Blood Genomic DNA Isolation Kit, Seoul, Korea). PCR amplification was performed using $50 \mathrm{ng}$ of genomic DNA in a $30 \mu \mathrm{l}$ reaction volume that contained $2.5 \mathrm{mM}$ dNTP $0.5 \mathrm{ml}, 2 \mathrm{U}$ of Taq DNA polymerase (Neurotics, Seoul, Korea), and PCR primer sets. Samples were subjected to 35 amplification cycles in GeneAmp PCR system 2700 (Applied Biosystems, Foster, CA). The PCR products were digested by PvuII (rs2234693) and $\mathrm{XbaI}$ (rs9340799) under conditions specified by the enzyme supplier (New England Biolabs, Beverly, MA). The PCR primers were sense strand 5 '-ctgccacctatctgatatctttcctattctcc$3^{\prime}$ and antisense strand 5'-tctttctctgccacctggcgtcgattatctga-3'. Restriction fragments were separated by agarose gel electrophoresis and ethidium bromide staining. The presence of the restriction site for each endonuclease was conventionally indicated with a lowercase letter ( $p$ or $x$, respectively, for PvuII and $\mathrm{XbaI}$ endonucleases), whereas the absence of the restriction site was indicated with a capital letter $(P$ or $X)$.

\section{Statistical analysis}

Multiple logistic regression analysis was used to estimate odds ratio (OR) and associated 95\% confidence interval (CI) for environmental factors. We conducted univariate analysis to identify significant covariates from the total study population that included medical and surgical cases. Age, education, number of years from menopause to enrollment time, past history of pulmonary tuberculosis, cancer history, hysterectomy, use of oral contraceptives, age at menarche, spontaneous abortion at first pregnancy (meaning whether a women's first pregnancy was successful or failed), and duration of breast feeding were selected as covariates in our analysis. In the analysis of idiopathic cases and controls, surgical or medical menopause-related variables such as hysterectomy, cancer history, and past history of pulmonary tuberculosis were excluded as adjustment variables.

The Hardy-Weinberg equilibrium assessed allele frequencies using the $\chi$-square test. Among POF cases, EM cases, and controls, genotype frequencies did not deviate from the Hardy-Weinberg equilibrium $(p>0.1)$. Moreover, single SNPs, haplotype, and diplotype analyses were performed. To detect each SNP effect of ESR1 for POF and EM, three genetic models-a codominant, dominant, and recessive model- 
were used, and the $p$-trend values derived from the additive model were presented. Tests for significance were computed after adjusting for age, education, number of years from menopause $(<10,>10$ year), smoking status, past history of pulmonary tuberculosis, age at menarche, spontaneous abortion at first pregnancy, and breastfeeding; all were selected from backward stepwise logistic regression.

Risks for POF and EM in relation to ESR1 haplotypes were assessed using SAS-Genetics software (version 8.2), ${ }^{32}$ which employs the expectation-maximization algorithm to estimate haplotypes. The XbaI and PvuII polymorphisms were in high linkage disequilibrium (Lewontin's D'value $=0.99$ ). A haplotype-based global score test assessed the overall differences in haplotype frequencies between POF or EM cases and NM controls. We assessed the haplotype-associated risks using the $\mathrm{P}-\mathrm{X}$ haplotype as the referent category after adjusting for age, education, number of years from menopause ( $\leq 10,>10$ year), smoking status, past history of pulmonary tuberculosis, age at menarche, spontaneous abortion at first pregnancy, and breastfeeding. The association between ESR1 haplotypes with observed frequencies greater than 5\% and POF and EM cases was evaluated. Diplotypes with at least one copy of the referent haplotype P-X were selected as the reference in the diplotype analysis. Risk of POF or EM was estimated for each diplotype compared to the referent diplotype, adjusting for the same covariates. The diplotype data were treated as categorical variables and were incorporated as dummy variables in the logistic regression models.

Polytomous logistic regression was used to estimate $p$ values for an association between genetic factors and the three menopausal groups (POF, EM, and NM) according to a nominal scale. Likelihood ratio tests for linear trend assessed a potential dose-response relationship. If any of the cell frequencies in a given table were less than three, we did not present the ORs and 95\% CIs.

\section{Results}

Compared to NM controls, EM cases were more educated and POF cases were less educated ( $p=0.01$ ) (data not shown). Table 1 presents the odds ratios and $95 \%$ CIs of environmental and reproductive risk factors such as smoking/alcohol status, physical activity, past history of pulmonary tuberculosis, age at menarche, parity, spontaneous abortion at first pregnancy, and breast feeding, for POF and EM. Past history of pulmonary tuberculosis and age at menarche were significant risk factors for both POF and EM (OR =1.6, 95\% CI 1.5-11.0 for $\mathrm{EM} ; \mathrm{OR}=5.0,95 \% \mathrm{CI} 1.5-16.4$ for POF in terms of past history of pulmonary tuberculosis; $\mathrm{OR}=4.1,95 \% \mathrm{CI} 1.5-11.0$ for EM; $\mathrm{OR}=5.6,95 \%$ CI 2.0-15.4 for POF in terms of age at menarche), but an increased risk was observed in POF cases. Breastfeeding for longer than 24 months was a significant protective factor for both POF and EM (OR $=0.3,95 \%$ CI 0.1 0.99 for $\mathrm{EM}$; OR = 0.2, 95\% CI 0.1-0.6 for POF). For idiopathic cases, age at menarche was significant for both EM and POF $(\mathrm{OR}=4.8,95 \%$ CI 1.7-14.0 for $\mathrm{EM}$; OR $=7.0,95 \%$ CI 2.2-23.0 for POF).

Table 2 shows the distribution of ESR1 polymorphisms among all cases and controls and the ORs (95\% CIs) for POF and EM cases compared to NM controls in relation to ESR1 genetic polymorphisms. Genotype frequencies for all SNPs did not deviate from the Hardy-Weinberg equilibrium $(p>0.1)$. In the single SNPs analysis, no significant allele effect was observed with the PvuII genotype. In contrast, The $\mathrm{XbaI}$ genetic variant containing the $\mathrm{X}$ allele was associated with a reduced risk for both total POF and idiopathic POF in the dominant model (OR = 0.6, 95\% CI 0.3-0.99 for total POF; $\mathrm{OR}=0.6,95 \%$ CI 0.3-1.0 for idiopathic POF). However, this $X$ allele effect was not observed for both total EM and idiopathic EM cases.

In the polytomous logistic regression, the dominant model showed that the $\mathrm{XbaI}$ genetic variant for POF risk was different from EM, relative to NM controls, and showed marginal significance $\left(p_{\text {polytomous }}=0.08\right)$. The $\mathrm{XbaI}$ genetic variant for idiopathic POF risk was also different from idiopathic EM $\left(p_{\text {polytomous }}=0.08\right)$. The two SNP-based global $p$ values were 0.17 for EM cases vs controls and 0.07 for POF cases vs controls for all cases including medical/surgical causes; and 0.39 for EM cases vs controls and 0.09 for POF cases vs controls for idiopathic causes.

Table 3 shows the association between haplotype-pairs of the ESR1 gene and POF and EM risk. In the haplotype analysis, three PvuII- XbaI haplotypes, P-X, P-x and $\mathrm{p}-\mathrm{X}$, were observed. The frequency of the $\mathrm{p}-\mathrm{x}$ haplotype was less than $2 \%$ in each group. The haplotype-based global $p$ value was 0.06 for EM cases vs controls and $<0.001$ for POF cases vs controls for all cases including medical/surgical causes; and 0.12 for EM cases vs controls and $<0.0001$ for POF cases vs controls for idiopathic causes.

Compared to the most frequent P-X haplotype, the P-x haplotype significantly decreased the risk of both total POF and idiopathic POF (OR $=0.5,95 \%$ CI 0.3-0.7 for total POF; $\mathrm{OR}=0.5,95 \%$ CI 0.2-0.9 for idiopathic POF). The $\mathrm{p}-\mathrm{X}$ haplotype also showed a decreased risk for both total POF and idiopathic POF but was not significant. In contrast to POF, the $\mathrm{P}-\mathrm{x}$ and $\mathrm{p}-\mathrm{X}$ haplotypes increased the risk for both EM and idiopathic EM but was not statistically significant. The P-x and $\mathrm{p}-\mathrm{X}$ haplotype effects were different for POF and EM and idiopathic POF and idiopathic EM. All were significant by polytomous regression analysis except the $\mathrm{p}-\mathrm{X}$ haplotype for idiopathic POF and EM ( $p_{\text {polytomous }}=0.0001, p_{\text {polytomous }}=0.009$, $p_{\text {polytomous }}=0.046$, respectively).

We recombined the six diplotypes into three groups and set the $\mathrm{P}-\mathrm{X}{ }^{*} \mathrm{P}-\mathrm{X}$ or $\mathrm{P}-\mathrm{X}^{*} \mathrm{p}-\mathrm{X}$ diplotypes as the reference group. Compared to the reference group, diplotypes that contained one or two copies of P-x statistically significantly decreased the risk of both total POF and idiopathic POF (OR $=0.4,95 \%$ CI 0.2-0.6 for total POF; OR = 0.4, 95\% CI 0.2-0.9 for idiopathic POF). Also, diplotypes with one or two copies of $\mathrm{p}-\mathrm{X}$ except $P-X^{*} p-X$ decreased the POF risk regardless of idiopathic type but were insignificant. One or two copies of the P-x diplotype was significantly different from POF and EM risk, regardless of idiopathic type $\left(p_{\text {polytomous }}=0.0001, p_{\text {polytomous }}=0.02\right)$ (Table 3).

\section{Discussion}

Our study showed that the ESR1 genetic variant was significantly associated with POF risk but not with EM. The $X$ allele of $\mathrm{XbaI}$ and specific haplo- and diplotype of PvuII and $\mathrm{XbaI}$ polymorphisms were associated with a significantly reduced risk of POF occurrence but not EM. This association remained in stratified analysis of only idiopathic cases. Moreover, results of nominal polytomous logistic regression 


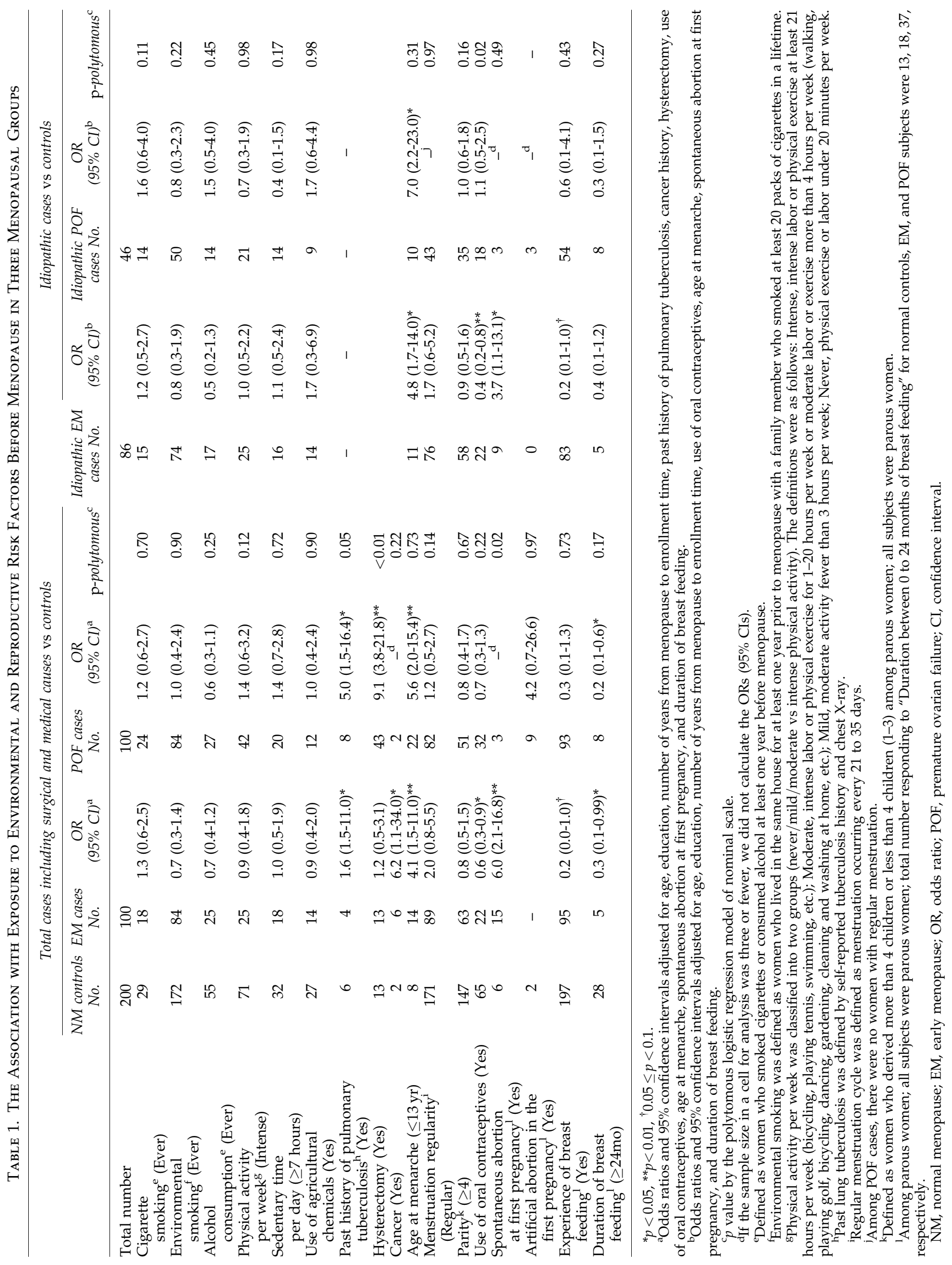




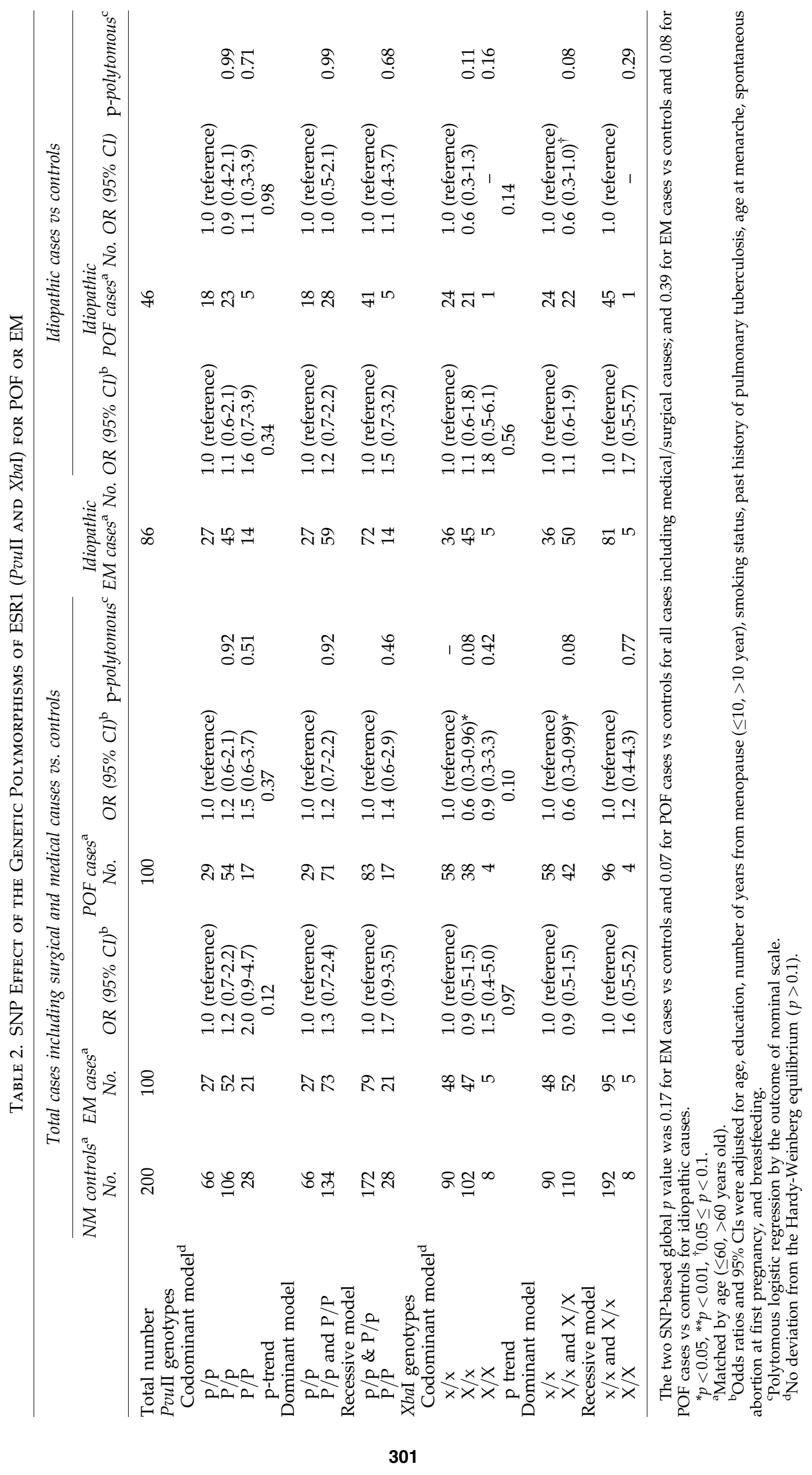




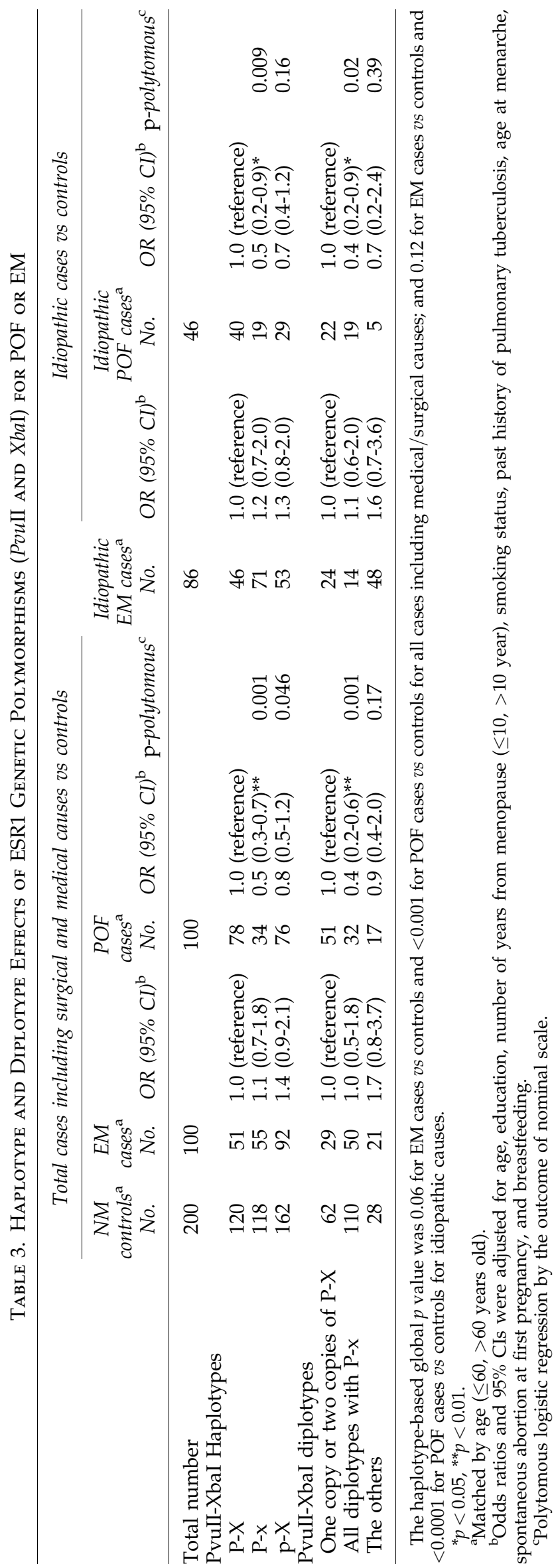

indicated that ESR1 genetic variants influence the etiology of POF and EM differently.

Previous studies that focused on the association of ESR1 $P v u I I$ or/and XbaI polymorphisms and the onset of menopause did not find a significant association. ${ }^{33-36}$ However, two studies reported that the ESR1 gene was significantly associated with the earlier onset of menopause. ${ }^{22,28}$ In one study, the homozygous $\mathrm{P}$ allele of $P v u \mathrm{II}$ was associated with 1.1 year earlier onset of menopause compared to the homozygous $\mathrm{p}$ allele. Also, an additive effect for each copy of the $\mathrm{P}$ allele was reported. ${ }^{22}$ Similarly, in our study, the homozygous $P$ allele of PvuII was associated with an increased risk for both POF and EM in the SNP effect, although statistically insignificant, possibly because of the small sample size. In another study, one of the ESR1 haplotypes, corresponding to the P-X haplotype, was associated with a significantly increased risk for POF. ${ }^{28}$ Concordant with this study, when the reference value was the P-x haplotype, the P-X haplotype was associated with a significantly increased risk for $\mathrm{POF}(\mathrm{OR}=2.2,95 \% \mathrm{CI} 1.4-3.7$ for total POF; OR $=2.2,95 \% \mathrm{CI}$ 1.2-4.2 for idiopathic POF).

Few studies have focused on ESR1 XbaI polymorphisms associated with age at menopause or POF. However, the $X$ allele of XbaI was reported to be related to increased bone mineral density, reduced risk of osteoporosis, and cardiovascular diseases, which suggest higher levels of estrogen. ${ }^{24,37-40}$ In terms of our study outcome, we can also infer that the $\mathrm{X}$ allele of $\mathrm{XbaI}$ may play a crucial role in protection against POF. However, we did not find evidence that the $X$ allele of $\mathrm{Xba \textrm {I }}$ is involved in EM development. Our study results, including our nominal polytomous logistic regression, suggest that development of POF and EM may be related to different genetic functions.

The biological pathway of XbaI and PvuII that relates to early onset of menopause is still unknown. An in vitro study reported that the P allele of PvuII may play a role in the amplification of ER- $\alpha$ transcription or may regulate the ESR1 expression and function. ${ }^{41}$ Although there is no information on the role of the XbaI X allele, the XbaI X allele could act with the PvuII P allele in ER- $\alpha$ transcription or ESR1 expression/

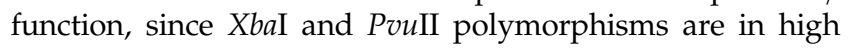
linkage disequilibrium.

When considering previous reports and the present study results, we hypothesize a series of mechanisms for POF and ER genetic polymorphisms: (1) Estrogen binds to ERs in reproductive tissues, such as the ovaries, uterus, and vagina. ${ }^{42}$ If the activity of ERs is low because of the low activity gene encoding protein, estrogenic action in the tissue may be weak; (2) Continuous weak estrogenic effect in the reproductive tissue, especially the ovaries, may have a negative feedback on the pituitary gland, especially follicle stimulating hormone (FSH) secretion; (3) FSH, in turn, can accelerate the rapid depletion of the ovarian follicles, leading to the development of POF because of ovarian dysfunction. To clarify the exact mechanisms between ER genes and POF and/or EM, additional studies are required.

Although, to our knowledge, this is the first study to report a genetic difference related to age at menopause, our study had several limitations. First, because of the high cost and low access to clinics and physicians in community populationbased survey settings, our POF cases were ascertained only by self-report, without FSH testing or other confirmation. Thus, 
we were not able to differentiate POF and premature menopause (PM) cases in our analysis. ${ }^{43}$ Second, our study included a small number of idiopathic POF and EM cases, so we did not have sufficient statistical power to observe a genegene or gene-environment interaction. Third, we genotyped only a small number of SNPs related to ERs, and thus we were not able to examine other important SNPs. Finally, we classified menopausal groups based on self-reported age at menopause, which is vulnerable to misclassification and recall bias. However, in order to minimize bias, we excluded subjects over the age of 60 and adjusted for the number of years from menopause.

In conclusion, our study shows that the ESR1 gene including PvuII and XbaI polymorphisms can modify the risk of idiopathic POF, but these variants are not associated with EM risk. This supports the possibility that the etiology of POF and EM may differ with regard to genetic factors. Further studies with sufficient POF and EM cases will help clarify the ESR1 genetic mechanism and examine the gene-gene and geneenvironment interactions.

\section{Disclosure Statement}

There is no conflict of interest that could be perceived as prejudicing the impartiality of the research reported.

\section{Acknowledgments}

This study was supported by grant (no. 02-PJ1-PG3-219040002) from the Korea Health 21 Research and Development Project, Ministry of Health and Welfare, Republic of Korea.

\section{References}

1. Kaprio J, Rimpelä A, Winter T, Viken RJ, Rimpelä M, Rose RJ. Common genetic influences on BMI and age at menarche. Hum Biol 1995;67:739-753.

2. Meyer JM, Eaves LJ, Heath AC, Martin NG. Estimating genetic influences on the age-at-menarche: a survival analysis approach. Am J Med Genet 1991;39:148-154.

3. Popat RA, Van Den Eeden SK, Tanner CM, et al. Effect of reproductive factors and postmenopausal hormone use on the risk of Parkinson disease. Neurology 2005;65:383-390.

4. Treloar SA, Martin NG. Age at menarche as a fitness trait: nonadditive genetic variance detected in a large twin sample. Am J Hum Genet 1990;47:137-148.

5. Wasserman L, Flatt SW, Natarajan L, et al. Correlates of obesity in postmenopausal women with breast cancer: comparison of genetic, demographic, disease-related, life history and dietary factors. Int J Obes Relat Metab Disord 2004;28:49-56.

6. Franceschi S, La Vecchia C, Booth M, et al. Pooled analysis of 3 European case-control studies of ovarian cancer: II. Age at menarche and at menopause. Int J Cancer 1991;49:57-60.

7. van der Schouw YT, van der Graaf Y, Steyerberg EW, Eijkemans JC, Banga JD. Age at menopause as a risk factor for cardiovascular mortality. Lancet 1996;347:714-718.

8. van der Klift $M$, de Laet $\mathrm{CE}$, McCloskey EV, et al. Risk factors for incident vertebral fractures in men and women: the Rotterdam Study. I Bone Miner Res 2004;19:11721180.

9. Bagur AC, Mautalen CA. Risk for developing osteoporosis in untreated premature menopause. Calcif Tissue Int 1992; 51:4-7.
10. Caplan GA, Scane AC, Francis RM. Pathogenesis of vertebral crush fractures in women. J R Soc Med 1994;87:200-202.

11. Palmer JR, Rosenberg L, Shapiro S. Reproductive factors and risk of myocardial infarction. Am J Epidemiol 1992;136: 408-416.

12. Snowdon DA, Kane RL, Beeson WL, et al. Is early natural menopause a biologic marker of health and aging? Am J Public Health 1989;79:709-714.

13. Nelson LM, Anasti JN, Flack MR. Premature ovarian failure. In: Adashi EY, Rock JA, Rosenwaks Z, eds. Reproductive endocrinology, surgery, and technology. Philadelphia: Lippincott-Raven. 1996:1394-1410.

14. Christin-Maitre S, Vasseur C, Portnoï MF, Bouchard P. Genes and premature ovarian failure. Mol Cell Endocrinol 1998;145:75-80.

15. Yan G, Schoenfeld D, Penney C, Hurxthal K, Taylor AE, Faustman D. Identification of premature ovarian failure patients with underlying autoimmunity. I Womens Health Gend Based Med 2000;9:275-287.

16. Laml T, Preyer O, Umek W, Hengstschlager M, Hanzal H. Genetic disorders in premature ovarian failure. Hum Reprod Update 2002;8:483-491.

17. Chang SH, Kim CS, Lee KS, et al. Premenopausal factors influencing premature ovarian failure and early menopause. Maturitas 2007;58:19-30.

18. de Bruin JP, Bovenhuis $H$, van Noord PA, et al. The role of genetic factors in age at natural menopause. Hum Reprod 2001;16:2014-2018.

19. Cramer DW, $\mathrm{Xu} \mathrm{H}$, Harlow BL. Family history as a predictor of early menopause. Fertil Steril 1995;64:740-745.

20. Torgerson DJ, Thomas RE, Reid DM. Mothers' and daughters' menopausal ages: is there a link? Eur J Obstet Gynecol Reprod Biol 1997;74:63-66.

21. Hsieh YY, Wang YK, Chang CC, Lin CS. Estrogen receptor alpha-351 $\mathrm{XbaI}^{*} \mathrm{G}$ and $-397 \mathrm{PvuII}^{*} \mathrm{C}$-related genotypes and alleles are associated with higher susceptibilities of endometriosis and leiomyoma. Mol Hum Reprod 2007;13: 117-122.

22. Weel AE, Uitterlinden AG, Westendorp IC, et al. Estrogen receptor polymorphism predicts the onset of natural and surgical menopause. J Clin Endocrinol Metab 1999;84:31463150 .

23. Kobayashi N, Fujino T, Shirogane T, et al. Estrogen receptor alpha polymorphism as a genetic marker for bone loss, vertebral fractures and susceptibility to estrogen. Maturitas 2002;41:193-201.

24. Ioannidis JP, Ralston SH, Bennett ST, et al. Differential genetic effects of ESR1 gene polymorphisms on osteoporosis outcomes. JAMA 2004;292:2105-2114.

25. Onland-Moret $\mathrm{NC}$, van Gils $\mathrm{CH}$, Roest $\mathrm{M}$, Grobbee DE, Peeters $\mathrm{PH}$. The estrogen receptor alpha gene and breast cancer risk (The Netherlands). Cancer Causes Control 2005; 16:1195-1202.

26. Kitamura I, Ando F, Koda M, Okura T, Shimokata H. Effects of the interaction between lean tissue mass and estrogen receptor alpha gene polymorphism on bone mineral density in middle-aged and elderly Japanese. Bone 2007;40:1623-1629.

27. Molvarec A, Vér A, Fekete A, et al. Association between estrogen receptor alpha (ESR1) gene polymorphisms and severe preeclampsia. Hypertens Res 2007;30:205-211.

28. Bretherick KL, Hanna CW, Currie LM, Fluker MR, Hammond GL, Robinson WP. Estrogen receptor alpha gene polymorphisms are associated with idiopathic premature ovarian failure. Fertil Steril 2008;89:318-324. 
29. Drummond AE, Findlay JK. The role of estrogen in folliculogenesis. Mol Cell Endocrinol 1999;151:57-64.

30. Kobayashi S, Inoue S, Hosoi T, Ouchi Y, Shiraki M, Orimo $\mathrm{H}$. Association of bone mineral density with polymorphism of the estrogen receptor gene. I Bone Miner Res 1996;11: 306-311.

31. Yoo KY, Shin HR, Chang SH, et al. Korean Multi-Center Cancer Cohort Study including a Biological Materials Bank (KMCC-I). Asian Pac J Cancer Prev 2002;3:85-92.

32. Excoffier L, Slatkin M. Maximum-likelihood estimation of molecular haplotype frequencies in a diploid population. Mol Biol Evol 1995;12:921-927.

33. Dvornyk V, Long JR, Liu PY, et al. Predictive factors for age at menopause in Caucasian females. Maturitas 2006;54: 19-26.

34. Gorai I, Tanaka K, Inada M, et al. Estrogen-metabolizing gene polymorphisms, but not estrogen receptor-alpha gene polymorphisms, are associated with the onset of menarche in healthy postmenopausal Japanese women. J Clin Endocrinol Metab 2003;88:799-803.

35. Kok HS, Onland-Moret NC, van Asselt KM, et al. No association of estrogen receptor alpha and cytochrome P450c17 alpha polymorphisms with age at menopause in a Dutch cohort. Hum Reprod 2005;20:536-542.

36. Schuit SC, de Jong FH, Stolk L, et al. Estrogen receptor alpha gene polymorphisms are associated with estradiol levels in postmenopausal women. Eur J Endocrinol 2005;153:327-334.

37. Lorentzon $M$, Lorentzon $R$, Bäckström T, Nordström P. Estrogen receptor gene polymorphism, but not estradiol levels, is related to bone density in healthy adolescent boys: a crosssectional and longitudinal study. J Clin Endocrinol Metab 1999;84:4597-4601.
38. Weiderpass E, Persson I, Melhus H, Wedrén S, Kindmark A, Baron JA. Estrogen receptor alpha gene polymorphisms and endometrial cancer risk. Carcinogenesis 2000;21:623-627.

39. van Meurs JB, Schuit SC, Weel AE, et al. Association of $5^{\prime}$ estrogen receptor alpha gene polymorphisms with bone mineral density, vertebral bone area and fracture risk. Hum Mol Genet 2003;12:1745-1754.

40. Schuit SC, Oei HH, Witteman JC, et al. Estrogen receptor alpha gene polymorphisms and risk of myocardial infarction. JAMA 2004;291:2969-2977.

41. Herrington DM, Howard TD, Brosnihan KB, et al. Common estrogen receptor polymorphism augments effects of hormone replacement therapy on E-selectin but not C-reactive protein. Circulation. 2002;105:1879-1882.

42. Pelletier G, El-Alfy M. Immunocytochemical localization of estrogen receptors alpha and beta in the human reproductive organs. J Clin Endocrinol Metab 2000;85:4835-4840.

43. Nelson LM, Anasti JN, Kimzey LM, et al. Development of luteinized graafian follicles in patients with karyotypically normal spontaneous premature ovarian failure. J Clin Endocrinol Metab 1994;79:1470-1475.

Address correspondence to: Sue K. Park, M.D., Ph.D. Department of Preventive Medicine Seoul National University College of Medicine,

28 Yeongeon-Dong, Jongro-Gu Seoul 110-799 Republic of Korea

E-mail: suepark@snu.ac.kr 


\section{This article has been cited by:}

1. Amanda Souza Setti, Sylvia Sanches Cortezzi, Rita de Cássia S. Figueira, Ciro Dresch Martinhago, Daniela Paes de Almeida Ferreira Braga, Assumpto Iaconelli, Edson Borges. 2012. A chromosome 19 locus positively influences the number of retrieved oocytes during stimulated cycles in Brazilian women. Journal of Assisted Reproduction and Genetics . [CrossRef]

2. Yingying Qin, Mei Sun, Li You, Deying Wei, Jielin Sun, Xiaoyan Liang, Bo Zhang, Hong Jiang, Jianfeng Xu, Zi-Jiang Chen. 2012. ESR1, HK3 and BRSK1 gene variants are associated with both age at natural menopause and premature ovarian failure. Orphanet Journal of Rare Diseases 7:1, 5. [CrossRef]

3. Sunshin Kim, Jung-A Pyun, HyunJun Kang, JiHye Kim, Dong Hyun Cha, KyuBum Kwack. 2011. Epistasis between CYP19A1 and ESR1 polymorphisms is associated with premature ovarian failure. Fertility and Sterility 95:1, 353-356. [CrossRef] 